\title{
Inhalation challenge in the differential diagnosis of usual interstitial pneumonia
}

\author{
To the Editor:
}

Establishing when a patient with usual interstitial pneumonia (UIP) is truly idiopathic is fundamental. In this sense, the paper recently published by WuYTs et al. [1], is an excellent review of the various diseases that may present with a histopathology consistent with UIP.

The authors stress the importance of distinguishing between idiopathic pulmonary fibrosis (IPF) and chronic hypersensitivity pneumonitis in the differential diagnosis, especially in view of the fact that immunosuppressant treatment is suitable for patients with hypersensitivity pneumonitis, but is clearly contraindicated in patients with IPF [2]. Furthermore, the authors note that these diagnoses can easily be confused; indeed, Morell et al. [3] recently found that up to $43 \%$ of patients diagnosed with IPF are actually misdiagnosed chronic hypersensitivity pneumonitis. As WuYTs et al. [1] state: "The diagnosis of hypersensitivity pneumonitis requires proven or suspected exposure associated with exposure-related symptoms; proof of sensitisation (e.g. demonstration of serum precipitins or lymphocytosis in the BAL fluid); and compatible chest radiography/HRCT abnormalities (with or without restrictive ventilatory/gas exchange defects) [4]. However, serum precipitating antibodies to a variety of common causative antigens have low sensitivity and specificity, and are helpful when positive but not if negative. Chronic hypersensitivity pneumonitis may represent a diagnostic dilemma, particularly when a detailed clinical history does not reveal a temporal relationship between symptom onset and antigen exposure". It is in this context that a specific inhalation challenge (SIC) may be particularly useful in the diagnosis of hypersensitivity pneumonitis, and also in order to differentiate it from IPF.

Indeed, of the 20 patients with hypersensitivity pneumonitis previously diagnosed as IPF reported by Morell et al. [3], SIC was essential in establishing differentiation in nine cases, although in eight of them precipitins were also positive. In the remaining 11 patients, the final diagnosis was made by combining exposure history, precipitins, lymphocytosis in bronchoalveolar lavage (BAL) and/or alterations in surgical lung biopsy showing a pattern consistent with chronic hypersensitivity pneumonitis. In this regard, our group has recently reported that, in patients with suspected hypersensitivity pneumonitis caused by exposure to avian proteins or fungi, SIC has a sensitivity of $85 \%$ and a specificity of $86 \%$, with a positive predictive value ranging from $84 \%$ to $97 \%$ and a negative predictive value from $55 \%$ to $84 \%$ [5].

Few centres use SIC for the diagnosis of hypersensitivity pneumonitis. In acute and subacute forms it is probably not essential for establishing a correct diagnosis, but in chronic forms, in which the differential diagnosis with IPF is crucial, it may well be helpful. In this context, in which the pathology is consistent with UIP, most authors agree that assessment of precipitins or of the presence of lymphocytosis in BAL are the most objective methods for determining whether or not the patient has hypersensitivity pneumonitis. However, it should be borne in mind that up to $60 \%$ of healthy subjects exposed to fungi may have positive precipitins [4] and up to 50\% of those exposed to birds as well [6]. However, between 10 and $15 \%$ of patients do not develop serum precipitins, so a negative finding does not rule out the presence of the disease [7]. In mould-induced hypersensitivity pneumonitis, the negative predictive values of serum antibodies were found to range from $81 \%$ to $88 \%$ and the positive predictive values from $71 \%$ to $75 \%$ [8], while in bird fancier's lung the negative predictive value ranged from $30 \%$ to $81 \%$ and the positive predictive value from $49 \%$ to $67 \%$ [9]. As for the presence of lymphocytosis in BAL, it has been proposed that BAL lymphocyte counts above $30 \%$ discriminate chronic hypersensitivity pneumonitis with a UIP pattern on high-resolution computed tomography from IPF [10]. However, not only does a figure below $30 \%$ not rule out the diagnosis, but some authors suggest that the cut-off point for suspecting the diagnosis should be lowered to $20 \%$ [9, 11]. It is also important to note that BAL lymphocyte counts may be lower in chronic fibrotic forms and may gradually decrease with cessation of exposure $[4,12]$. Therefore, the need for improved diagnostic methods is clear.

Table 1 shows the unpublished data from a recent study by our group evaluating the utility of SIC for diagnosing hypersensitivity pneumonitis [6]. Significantly, 29 patients with positive SIC who were diagnosed with hypersensitivity pneumonitis had a BAL lymphocyte count below 20\%. Similar data have also been observed by OHTANi et al. [11]. In 18 patients in this group (positive SIC and positive for hypersensitivity pneumonitis) precipitins were negative. Table 1 also shows the combination of positive 
TABLE 1 Specific IgG and percentage of lymphocytes in BAL in patients with suspected HP in whom a SIC was performed

\begin{tabular}{|c|c|c|c|c|}
\hline & $\begin{array}{c}\text { Group } 1 \\
\left(\mathrm{HP}^{+}, \mathrm{SIC}^{+}\right)\end{array}$ & $\begin{array}{c}\text { Group } 2 \\
\left(\mathrm{HP}^{-}, \mathrm{SIC}^{+}\right)\end{array}$ & $\begin{array}{c}\text { Group } 3 \\
\left(\mathrm{HP}^{+}, \mathrm{SIC}^{-}\right)\end{array}$ & $\begin{array}{c}\text { Group } 4 \\
\left(H P^{-}, \mathrm{SIC}^{-}\right)\end{array}$ \\
\hline Subjects & 64 & 4 & 24 & 21 \\
\hline \multicolumn{5}{|l|}{ Antigen } \\
\hline Moulds & 23 & 2 & 4 & 3 \\
\hline Avian & 34 & 2 & 17 & 13 \\
\hline Others & 7 & 0 & 3 & 5 \\
\hline \multicolumn{5}{|l|}{ Specific IgG } \\
\hline Positive & 39 & 2 & 11 & 9 \\
\hline Negative & 18 & 2 & 8 & 7 \\
\hline Not done & 7 & 0 & 5 & 5 \\
\hline \multicolumn{5}{|c|}{ Lymphocytes in BAL \% } \\
\hline$<20$ & 29 & 3 & 8 & 9 \\
\hline $20-30$ & 5 & 0 & 5 & 3 \\
\hline$>30$ & 14 & 1 & 4 & 0 \\
\hline Not done & 16 & 0 & 7 & 9 \\
\hline \multicolumn{5}{|c|}{ Specific IgG ${ }^{+}$and $\%$ lymphocytes in BAL } \\
\hline$<20$ & 23 & 1 & 6 & 5 \\
\hline $20-30$ & 5 & 0 & 1 & 1 \\
\hline$>30$ & 12 & 1 & 3 & 0 \\
\hline \multicolumn{5}{|c|}{ Specific $\lg G^{-}$and $\%$ lymphocytes in BAL } \\
\hline$<20$ & 4 & 2 & 2 & 4 \\
\hline $20-30$ & 0 & 0 & 1 & 1 \\
\hline$>30$ & 2 & 0 & 1 & 0 \\
\hline
\end{tabular}

Data are presented as $\mathrm{n}$, unless otherwise stated. Data correspond to the study population in [5]. BAL: bronchoalveolar lavage; HP: hypersensitivity pneumonitis; SIC: specific inhalation challenge.

specific IgGs and BAL lymphocyte count. Only six patients with negative IgG and BAL lymphocyte count below $20 \%$ were diagnosed with hypersensitivity pneumonitis. Four of them had a positive SIC and two were negative. We believe that these data stress the usefulness of SIC in the differential diagnosis of chronic hypersensitivity pneumonitis and IPF.

As our group recently demonstrated, SIC in the context of hypersensitivity pneumonitis is a safe procedure with few side-effects and does not generate sensitisation [5]. The infrequency of its use is probably due to a lack of standardisation at the methodological level (i.e. with regard to the preparation of extracts, the form and quantity of inhalation), and the interpretation of results.

We hope that the different scientific societies will take steps to standardise and disseminate this technique. In our view, it has a vital role to play in the evaluation of chronic interstitial pneumonias, given that as many as $50 \%$ of cases diagnosed as IPF may be chronic hypersensitivity pneumonitis which has gone unnoticed.

Specific inhalation challenge is useful for the differential diagnosis of usual interstitial pneumonia http://ow.ly/PX1q9

Xavier Muñoz ${ }^{1,2,3}$, Monica Sanchez-Ortíz ${ }^{1,2}$, Inigo Ojanguren ${ }^{1}$ and María Jesús Cruz ${ }^{1,2}$

${ }^{1}$ Servei de Pneumologia, Hospital General Valld'Hebron, Departament de Medicina, Universitat Autonoma de Barcelona, Barcelona, Spain. ${ }^{2}$ CIBER Enfermedades Respiratorias (CIBERES), Instituto de Salud Carlos III, Barcelona, Spain. ${ }^{3}$ Departamento de Biología Celular, Fisiología e Inmunología, Universitat Autonoma de Barcelona, Barcelona, Spain.

Correspondence: Xavier Muñoz, Servei de Pneumologia, Hospital General Valld'Hebron, PasseigValld'Hebron, 119, 08035 Barcelona, Spain. E-mail: xmunoz@vhebron.net

Received: Jan 142015 | Accepted: Jan 252015

Support statement: M.J. Cruz is a researcher supported by the Miguel Servet programme from Instituto de Salud Carlos III (CP12/03101).

Conflict of interest: None declared.

Provenance: Submitted article, peer reviewed. 


\section{References}

1 Wuyts WA, Cavazza A, Rossi G, et al. Differential diagnosis of usual interstitial pneumonia: when is it truly idiopathic? Eur Respir Rev 2014; 23: 308-319.

2 Idiopathic Pulmonary Fibrosis Clinical Research Network, Raghu G, Anstrom KJ, et al. Prednisone, azathioprine, and $N$-acetylcysteine for pulmonary fibrosis. N Engl J Med 2012; 366: 1968-1977.

3 Morell F, Villar A, Montero MA, et al. Chronic hypersensitivity pneumonitis in patients diagnosed with idiopathic pulmonary fibrosis: a prospective case-cohort study. Lancet Respir Med 2013; 1: 685-694.

4 Costabel U, Bonella F, Guzman J. Chronic hypersensitivity pneumonitis. Clin Chest Med 2012; 33: 151-163.

5 Muñoz X, Sánchez-Ortiz M, Torres F, et al. Diagnostic yield of specific inhalation challenge in hypersensitivity pneumonitis. Eur Respir J 2014; 1658-1665.

6 Rodrigo MJ, Benavent MI, Cruz MJ, et al. Detection of specific antibodies to pigeon serum and bloom antigens by enzyme linked immunosorbent assay in pigeon breeder's disease. Occup Environ Med 2000; 57: 159-164.

7 Cormier Y, Bélanger J. The fluctuant nature of precipitating antibodies in dairy farmers. Thorax 1989; 44: 469-473.

8 Fenoglio CM, Reboux G, Sudre B, et al. Diagnostic value of serum precipitins to mould antigens in active hypersensitivity pneumonitis. Eur Respir J 2007; 29: 706-712.

9 Morell F, Roger A, Reyes L, et al. Bird fancier's lung: a series of 86 patients. Medicine 2008; 87: 110-130.

10 Ohshimo S, Bonella F, Cui A, et al. Significance of bronchoalveolar lavage for the diagnosis of idiopathic pulmonary fibrosis. Am J Respir Crit Care Med 2009; 179: 1043-1047.

11 Ohtani Y, Saiki S, Kitaichi M, et al. Chronic bird fancier's lung; histopathological and clinical correlation. An application of the 2002 ATS/ERS consensus classification of the idiopathic interstitial pneumonias. Thorax 2005; 60: 665-671.

12 Costabel U, Bross KJ, Marxen J, et al. T-lymphocytosis in bronchoalveolar lavage fluid of hypersensitivity pneumonitis. Changes in profile of T-cell subsets during the course of disease. Chest 1984; 85: 514-522. 